\title{
On the size distribution of ice-supersaturated regions in the upper troposphere and lowermost stratosphere
}

\author{
K. Gierens, P. Spichtinger ${ }^{1}$ \\ Deutsches Zentrum für Luft- und Raumfahrt, Institut für Physik der Atmosphäre, Oberpfaffenhofen, D-82234 Weßling, Germany \\ E-mail: klaus.Gierens@dlr.de
}

Received: 1 October 1999 / Revised: 13 December 1999 / Accepted: 1 January 2000

\begin{abstract}
In order to determine typical sizes of icesupersaturated regions (ISSRs) in the upper troposphere and lowermost stratosphere we set up the frequency distribution of path lengths flown by MOZAIC aircraft within ISSRs. The mean path length is about $150 \mathrm{~km}$ with a standard deviation of $250 \mathrm{~km}$. We analyse the influence of a selection bias (viz. that large ISSRs are more often crossed by aircraft than small ones) on the obtained path length statistics and derive a mathematical equation that relates the path length distribution to the underlying size distribution of ISSRs, assuming that they have circular shape. We solve the equation (by trial and error) and test the result using numerical simulations. Surprisingly, we find that there may be many more very small ISSRs than apparent from the data such that the true mean diameter of the ISSRs may be of the order a few kilometres only. The relevance of the result is discussed and dedicated research flights to measure the true extension of ISSRs are recommended.
\end{abstract}

Key words: Atmospheric composition and structure (troposphere - composition and chemistry; general)

\section{Introduction}

There is now direct evidence, obtained from the MOZAIC project (Marenco et al., 1998), that ice-supersaturation is frequent in upper tropospheric clear air and that it does even occur in the lowermost stratosphere (Gierens et al., 1999). In fact $13.5 \%$ of the more than 1.7 million MOZAIC data records analysed by Gierens et al. (1999) imply ice-supersaturation.

\footnotetext{
Correspondence to: K. Gierens

${ }^{1}$ Present address: Mathematisches Institut der Universität, Augsburg, Germany
}

Due to the scarcity of deposition nuclei (which would allow heterogeneous ice nucleation directly from the water phase) a small degree (some percent) of icesupersaturation is usually not sufficient to form a visible cirrus cloud. Instead, the most common cirrus formation pathway seems to be homogeneous freezing of solution droplets (e.g., Sassen and Dodd, 1989; Heymsfield and Miloshevich, 1993; Ström et al., 1997) which requires substantial supersaturation. Employing the empirical formula given by Heymsfield et al. (1998), Gierens et al. (1999) derived for the MOZAIC data, an average of $30 \%$ ice-supersaturation necessary for cirrus formation, whereas the mean supersaturation was only about $15 \%$. Furthermore, the probability of a certain supersaturation turned out to decrease exponentially with supersaturation which makes cirrus formation in such airmasses rather improbable. Persistent aircraft contrails are the only type of visible clouds that can be found in such ice-supersaturated regions (ISSRs).

Gierens et al. (1999) considered the frequency of occurrence of ISSRs, their thermodynamic properties in contrast to subsaturated regions, and discussed physical processes that would lead to the observed humidity distribution laws. In the present study we want to analyse the horizontal spatial extension of ISSRs, as far as it is possible with data obtained from commercial flights. In the next section we obtain a frequency distribution of path lengths flown inside ISSRs by the MOZAIC aircraft. In Sect. 3 we discuss how the measured path length distribution can be affected by the fact that large ISSRs have a larger probability of being crossed by aircraft than small ISSRs. We will derive a mathematical equation that relates the measured apparent size distribution of ISSRs to the underlying "true" size distribution (the meaning of "true" will become clear later). Unfortunately, there is at present no method to solve the equation, either analytically or numerically. Thus we sought a solution by trial and error. The result found in this unpleasing way has been tested by numerical simulation and its relevance is discussed in Sect. 4. Conclusions are drawn in Sect. 5. 


\section{Data evaluation}

As in our previous paper (Gierens et al., 1999) we have used three years of MOZAIC data (January 1995December 1997), constrained to the pressure range 175$275 \mathrm{hPa}$. This pressure range means upper troposphere and lowermost stratosphere. The flights took place mainly on the air routes between Europe and North America. This implies that the results refer mainly to the North Atlantic flight corridor. The geographical distribution of MOZAIC flights is given in Marenco et al. (1998, their Fig. 5) or on the MOZAIC home page (//www.cnrm.meteo.fr:8000/mozaic/). We use $1 \mathrm{~min}$ averages of only temperature and relative humidity in order to determine whether the aircraft flew in icesupersaturated air $(I C=1)$ or not $(I C=0)$. One minute of flight time corresponds to roughly $15 \mathrm{~km}$ flight distance. A sampling interval of $15 \mathrm{~km}$ is sufficient for a smooth field like the binary quantity $I C$ which we consider here. The smoothness of the $I C$ field becomes evident when one looks at persistent contrails (persistence needs $I C=1$ ), which are long and continuous stripes and not intermittently broken lines. For each of the 5269 flights considered we count the number of consecutive data records with $I C=1$ and determine the respective distance flow in ice-supersaturated air, $L$, by multiplying the result with $L_{0}=15 \mathrm{~km}$. The (nonnormalised) statistical distribution of these distances, $N(L)$, is plotted in Fig. 1.

The mean distance MOZAIC aircraft flew in icesupersaturated air is about $150 \mathrm{~km}$ with a standard deviation of about $250 \mathrm{~km}$. These values are consistent with the results of an earlier study by Detwiler and Pratt (1984), who, however, had a coarse spatial resolution of $75 \mathrm{~km}$ and a rather small data base. A spatial scale of $150 \mathrm{~km}$ is also in agreement with the length scales of condensation trail clusters as seen from satellites (Bakan et al., 1994; Mannstein et al., 1999). The appearance of

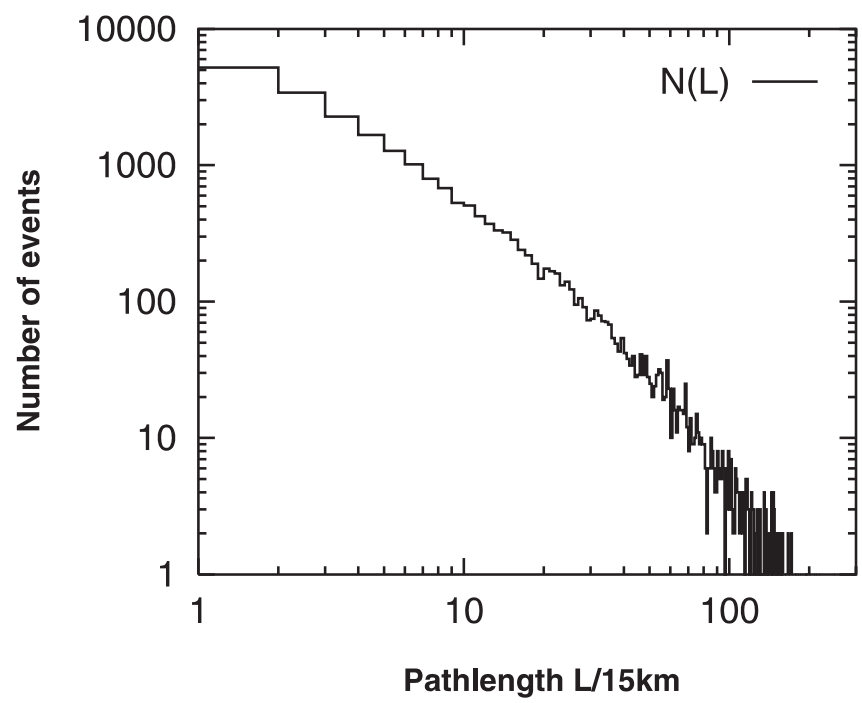

Fig. 1. Non-normalised statistical distribution, $N(L)$, of path lengths flown by MOZAIC aircraft within ice-supersaturated regions in the upper troposphere and lowermost stratosphere on flights between 1995 and 1997 contrails in satellite imagery is a safe indicator for icesupersaturation.

The longest distance flown in ice-supersaturated air occurred on a flight from Frankfurt (Germany) to an airport near Rio de Janeiro (Brazil) on December 18, 1995. This distance was $3735 \mathrm{~km}$ (i.e. 249 consecutive data records with $I C=1$ ). The ISSR, that this flight occurred in, was an uplifting airmass before a cold front that approached the northwestern African coast. The flight direction was almost parallel to the front line which explains the long extension of the ISSR. The region directly adjacent to the front where the flight occurred was clear (as can be seen from the satellite pictures of this day). However, further away from the front, over the African continent, the uplifting had already led to cloud formation. Huge ice-supersaturated regions can exist even in the lowermost stratosphere; the longest distance there occurred on a flight from Osaka (Japan) to Vienna (Austria) via the polar route on March 21, 1997, along the northern coast of Siberia (see Gierens et al., 1999).

\section{Mathematical analysis}

We must be aware of the fact that the MOZAIC aircraft are commercial airliners, and that they cross icesupersaturated regions (ISSR) by chance, not intention. Since it is more probable to encounter large ISSR than a small one by chance, there is a selection bias. In other words, the measured distribution of distances flown inside ISSR is only an apparent ISSR size distribution, that may differ substantially from the true ISSR size distribution. In the following we present a mathematical analysis of the problem, and we try to determine the "true" ISSR size distribution under the assumption that ISSR have circular shapes. This simplification is necessary in order to keep the problem tractable with only one degree of freedom, namely the circle diameter, $D$. More complicated shapes mean more degrees of freedom which renders the problem intractable. ISSRs of different sizes could have different lifetimes which would then add another bias to the evaluation of the path length data. However, this additional complication has not been taken into account in the following analysis since the MOZAIC data, by the very nature of their acquisition, do not allow any statements about the evolutionary stage or lifetime of the crossed ISSRs.

\subsection{The relation between true and apparent sizes for circular ISSRS}

Let us assume that an aircraft heading into direction $y$ crosses a circular ISSR of diameter $D$ at a random $x$-coordinate $u D / 2$ (see the sketch Fig. 2).

Then the distance $L$ flown inside the ISSR is:

$L=D \cdot \sqrt{1-u^{2}} \quad$ with $u \in[-1,+1]$ and $u=\frac{2 x}{D}$.

The normalised coordinate $u$ is uniformly distributed between -1 and +1 , so that its probability density is 


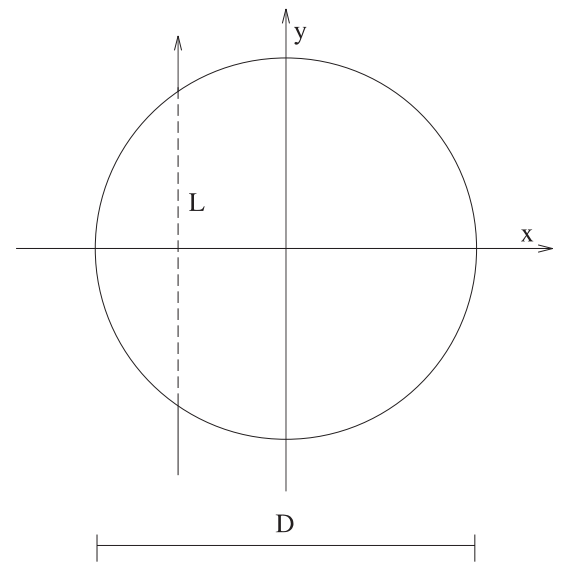

Fig. 2. Geometrical sketch for the derivation of Eq. (5)

$$
g(u) \mathrm{d} u=\frac{1}{2} \mathrm{~d} u,
$$

and the corresponding (cumulative) distribution function is

$G(u)=\frac{1}{2}(u+1)$.

Let us assume for the moment that all circular ISSRs have the same diameter $D$, then we obtain the corresponding distribution function, $F_{D}(L)$, in the following way $(P\{\cdot\}$ means probability for the event given in the brackets):

$$
\begin{aligned}
F_{D}(L) & =P\{l \leq L\}=P\left\{u^{2} \geq 1-L^{2} / D^{2}\right\} \\
& =P\left\{u \geq+\sqrt{1-L^{2} / D^{2}}\right\}+P\left\{u \leq-\sqrt{1-L^{2} / D^{2}}\right\} \\
& =1-G\left(+\sqrt{1-L^{2} / D^{2}}\right)+G\left(-\sqrt{1-L^{2} / D^{2}}\right) \\
& =1-\sqrt{1-L^{2} / D^{2}} .
\end{aligned}
$$

The corresponding probability density, $f_{D}(L)$, is the derivative of $F_{D}(L)$ :

$f_{D}(L)=\frac{L}{D} \frac{1}{\sqrt{D^{2}-L^{2}}}$.

Now we proceed to the general case of circular ISSRs with diameters distributed according to a (still unknown) function $h(D)$. The selection bias mentioned already, i.e. that it is more probable to hit large ISSRs than small ones, is accounted for by an additional factor $\frac{D}{\langle D\rangle}$, where $\langle D\rangle$ is the expectation value of $D$ with respect to $h(D)$. An aircraft approaching a circular ISSR "sees" the projection of the circle, i.e. its diameter $D$, not its area. Thus it is reasonable to assume a correction factor that is proportional to $D$, not to $D^{2}$. Then the probability density, $f(L)$, for distances flown through ISSRs of various sizes is given by the following basic equation:

$$
\begin{aligned}
f(L) & =\frac{1}{\langle D\rangle} \int_{L}^{\infty} f_{D}(L) D \cdot h(D) \mathrm{d} D \\
& =\frac{L}{\langle D\rangle} \int_{L}^{\infty} \frac{h(D) \mathrm{d} D}{\sqrt{D^{2}-L^{2}}}=: T[h(D)],
\end{aligned}
$$

where we have already introduced the operator notation, $T[\cdot]$, for later use.

The moments of a probability density $f_{X}(x)$ of the random variable $X$ are defined as follows

$\mu_{r}[X]:=\int_{0}^{\infty} x^{r} f_{X}(x) \mathrm{d} x$,

where $\mu_{1}[X]=\langle X\rangle$ is the expectation value of $X$, especially

$\langle D\rangle=\int_{0}^{\infty} D h(D) \mathrm{d} D$.

The moments $\mu_{r}[L]$ and $\mu_{r}[D]$ are related in the following way $(\Gamma(\cdot)$ is the gamma function):

$$
\begin{aligned}
\mu_{r}[L] & =\int_{0}^{\infty} L^{r} f(L) \mathrm{d} L \\
& =\int_{0}^{\infty} \frac{L^{r+1}}{\langle D\rangle} \int_{L}^{\infty} \frac{h(D) \mathrm{d} D}{\sqrt{D^{2}-L^{2}}} \mathrm{~d} L \\
& =\frac{1}{\langle D\rangle} \int_{0}^{\infty} \int_{0}^{D} \frac{L^{r+1} \mathrm{~d} L}{\sqrt{D^{2}-L^{2}}} h(D) \mathrm{d} D \\
& =\frac{1}{\langle D\rangle} \int_{0}^{\infty} \frac{\sqrt{\pi} \Gamma\left(\frac{2+r}{2}\right)}{2 \Gamma\left(\frac{3+r}{2}\right)} D^{r+1} h(D) \mathrm{d} D \\
& =\frac{\sqrt{\pi}}{2\langle D\rangle} \frac{r}{r+1} \frac{\Gamma\left(\frac{r}{2}\right)}{\Gamma\left(\frac{r+1}{2}\right)} \mu_{r+1}[D] .
\end{aligned}
$$

This means in particular:

$\mu_{1}[L]=\frac{\pi}{4\langle D\rangle} \mu_{2}[D]$,

$\mu_{2}[L]=\frac{2}{3\langle D\rangle} \mu_{3}[D]$,

and for the variance and the standard deviation we have:

$$
\begin{aligned}
\operatorname{Var}[L] & =\mu_{2}[L]-\mu_{1}[L]^{2} \\
& =\frac{2}{3\langle D\rangle} \mu_{3}[D]-\frac{\pi^{2}}{16\langle D\rangle^{2}} \mu_{2}[D]^{2}, \\
\sigma_{L}= & \sqrt{\operatorname{Var}[L]}=\sqrt{\frac{2}{3\langle D\rangle} \mu_{3}[D]-\frac{\pi^{2}}{16\langle D\rangle^{2}} \mu_{2}[D]^{2}} .
\end{aligned}
$$

\subsection{Determination of the "true" size distribution}

We are faced with the mathematical problem of how to derive the density $h(D)$ given the density $f(L)$ (which is the normalised version of $N(L)$ ). The relation between these two densities, Eq. (6) is unfortunately a one-way relation: The determination of $f(L)$ given $h(D)$ is straightforward, but there is at present no mathematical 
method to solve the inverse problem we are faced with, that is to find the inverse, $T^{-1}$, of the operator $T$. In fact, it is even unclear whether an inverse operator exists at all and whether $h(D)$, given $f(L)$, is unique. Thus we have tried to find a function $h(D)$ given $f(L)$ by trial and error, i.e. we try a function $h(D)$, evaluate the integral in Eq. (6) and test whether the resulting $f(L)$, scaled properly, provides a good fit to the data $N(L)$, i.e. the non-normalised empirical path length distribution given by the MOZAIC data.

Unfortunately, to insert for $h(D)$ common distributions like beta, exponential, gamma, or Weibull does not yield satisfactory results. Instead it was necessary to assume a generalised gamma distribution for $h(D)$. The resulting intricate integral could be solved with the MATHEMATICA software, and the result for $f(L)$ is a generalised Meijer's G-function which fits $N(L)$ (after scaling) reasonably well. (Note that the more common test distributions lead to similar cumbersome functions).

The generalised gamma density is given as

$\gamma(x, b, d, a):=\frac{1}{a^{d} \Gamma\left(\frac{d}{b}\right)} b x^{d-1} \mathrm{e}^{-\left(\frac{x}{a}\right)^{b}}$.

Inserting this for $h(D)$ in Eq. (6), and chosing the special case $b=1 / 6, d=1$, we can solve the integral analytically:

$$
\begin{aligned}
& T\left[\gamma\left(x, \frac{1}{6}, 1, a\right)\right]=\frac{L}{2554675200 \sqrt{6} a^{2} \pi^{5}} . \\
& \quad \times H_{0,12}^{12,0}\left(\frac{1}{12}\left(\frac{L}{a}\right),\left.\frac{1}{12}\right|_{0,0, \frac{1}{12}, \frac{2}{2}, \frac{3}{12}, \frac{4}{2}, \frac{5}{12}, \frac{7}{2}, \frac{8}{12}, \frac{9}{12}, \frac{10}{12}, \frac{11}{12}}\right),
\end{aligned}
$$

where $H$ is the generalised Meijer's G-function which is defined as follows:

$$
\begin{aligned}
& H_{p, q}^{m, n}\left(z, r \mid \begin{array}{l}
a_{1} \ldots a_{p} \\
b_{1} \ldots b_{q}
\end{array}\right) \\
& \quad:=\frac{r}{2 \pi i} \int_{C} \frac{\prod_{j=1}^{m} \Gamma\left(b_{j}-s r\right) \prod_{j=1}^{n} \Gamma\left(1-a_{j}+s r\right) z^{s} \mathrm{~d} s}{\prod_{j=m+1}^{q} \Gamma\left(1-b_{j}+s r\right) \prod_{j=n+1}^{p} \Gamma\left(a_{j}-s r\right)},
\end{aligned}
$$

where $C$ is an appropriate integration path around the poles (see Mathai, 1993). The expression for Meijer's function is somewhat more comfortable in our special case, viz.

$H_{0,12}^{12,0}\left(r,\left.z\right|_{b_{1} \ldots b_{12}}\right)=\frac{r}{2 \pi i} \int_{C} \prod_{j=1}^{12} \Gamma\left(b_{j}-s r\right) z^{s} \mathrm{~d} s$.

A reasonably good fit to $N(L)$ is achieved with $a=1.25 \cdot 10^{-6}$ and a scaling factor $\mathscr{N}=72000$, as shown in Fig. 3 or with slightly different parameters, e.g. $a=1.2 \cdot 10^{-6}$ and $\mathscr{N}=75000$ (Fig. 4). We did not try to minimise least squares because we deemed the necessary effort not worth while regarding the crudeness of our underlying assumption of circular shapes of

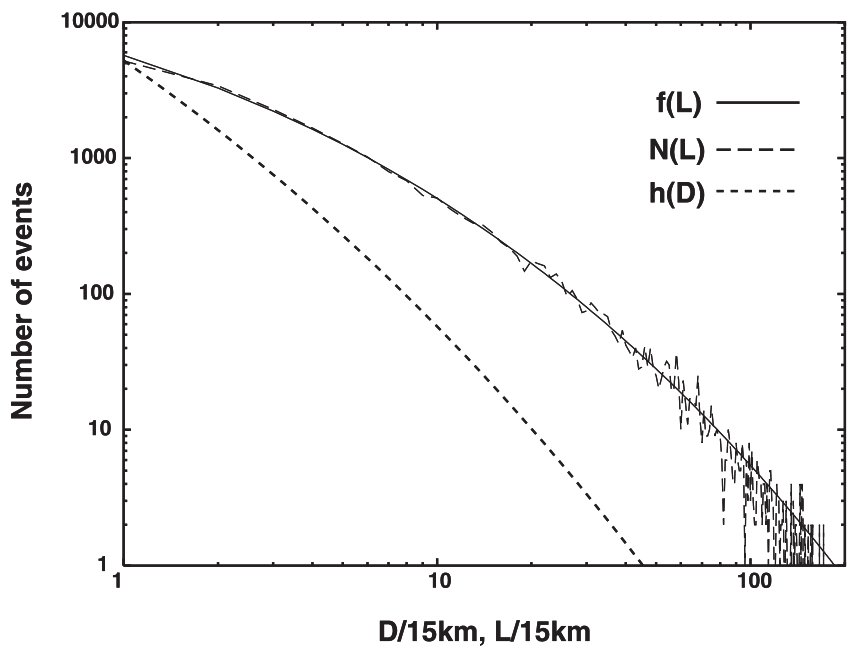

Fig. 3. As Fig. 1, together with a "true" size distribution, $h(D)$, of circular ice-supersaturated regions, and a resulting path length distribution, $f(L)$, that fits the original path length distribution $N(L)$ reasonably well. $h(D)$ is a generalised gamma-distribution with parameters $a=0.00000125, d=1, b=1 / 6 . f(L)$ was scaled with a factor $\mathscr{N}=72000$ to fit $N(L)$

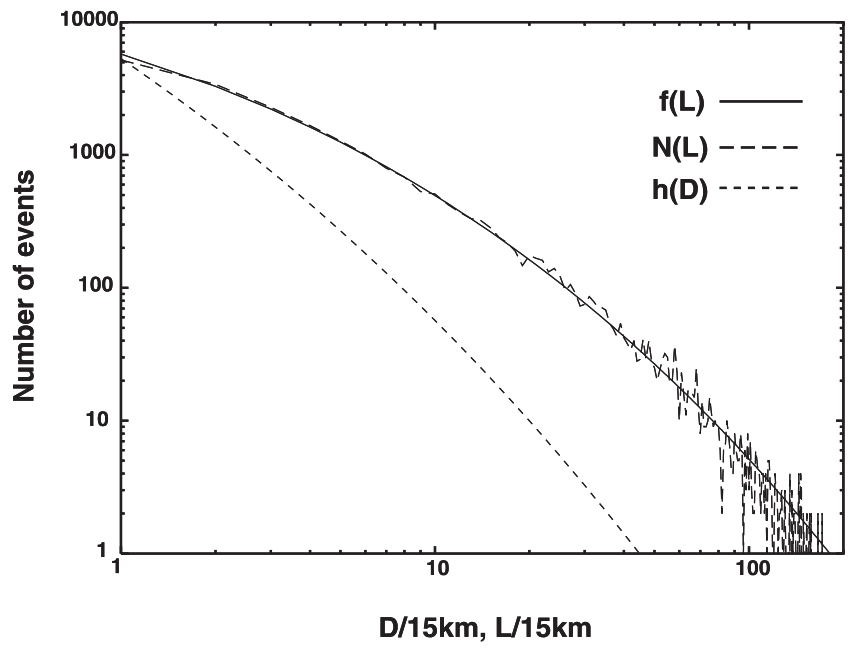

Fig. 4. As Fig. 3, but with the parameters: $a=0.0000012$, $\mathscr{N}=75000$

ISSRs. The resulting moments, mean values and standard deviations for $D$ and $L$ are given in Table 1.

It is remarkable that the "true" mean value of the diameters of the circular ISSRs is a factor of about 20 smaller than the mean distance the MOZAIC aircraft flew in ice-supersaturated air. The mean diameter is even smaller than the $15 \mathrm{~km}$ resolution of the MOZAIC data. This shows that the measured apparent ISSR sizes may be heavily biased towards the larger of these regions. Small ISSRs could be much more frequent than it appears from the aircraft data. We believe that this surprising result stays true when the actual shapes of ISSRs would (could) be taken into account, since the assumption of increasing probability of being crossed by an aircraft with increasing ISSR size stays valid also for 
Table 1. Mean values $\langle L\rangle$ and standard deviations $\sigma_{L}$ of path length distribution $N(L)$ for flights through ice-supersaturated regions according to MOZAIC data and two fits according to the mathematical model described in the text. The mean "true" diameters, $\langle D\rangle$, of the assumed circular ice-supersaturated regions and the corresponding standard deviations $\sigma_{D}$ are also given. All quantities are in units of kilometres

\begin{tabular}{lllll}
\hline Data & $\langle L\rangle$ & $\sigma_{L}$ & $\langle D\rangle$ & $\sigma_{D}$ \\
\hline MOZAIC path lengths & 150 & 250 & - & - \\
Fit with $a=1.20 \times 10^{-6}$ & 126 & 352 & 6.0 & 30 \\
Fit with $a=1.25 \times 10^{-6}$ & 131 & 367 & 6.2 & 32 \\
\hline
\end{tabular}

general shapes of ISSRs. However, the mathematical derivation just presented is not a proof that there are many small ISSRs. It could be that there are only few small ISSRs. Since it is not possible so far to analytically derive a solution to Eq. 6 we cannot be sure whether there is another solution $h(D)$ with fewer small diameters that would yield an equally good fit to that found by trial and error.

\section{Discussion}

\subsection{Numerical tests}

We have performed two numerical simulations in order to test the analytical result. First, we constructed a set of circles with diameters distributed according to the generalised gamma-density. These circles were placed at random positions but avoiding overlapping onto a discrete 2D grid with fine meshes, until the domain was filled up to about $15 \%$ of its area. A grid matrix $A=\left(a_{x y}\right)$ was introduced to characterise the position of the circles on the domain. Meshes inside a circle were given an indicator value $a_{x y}=1$ whereas outside the circles the grid matrix $A=\left(a_{x y}\right)$ contained zeroes. We then simulated flights by walking along $y$-axes and simulated the measurement of path lengths inside ISSRs by counting consecutive entries $a_{x y}=1$ along $y$-axes. We followed either all $y$-axes, or we chose them randomly. The result was the same (but for some statistical noise).

In a second test we used the grid matrix in a different way: all entries are zeroes except for grid points that were chosen as midpoints of the circles. The entries for these gridpoints were the diameters of the corresponding circles, i.e. $a_{x y}=D$. The simulation of the flights was as before, but the path lengths $L$ were now computed using formula 1 . The result of this test was not statistically different from the results of the other simulation. The simulated distribution of path lengths of both tests together with the original data $N(L)$ are plotted in Fig. 5. Aside from noise there is no systematic difference between the original data and the simulated data. This means that circular ISSRs with diameters distributed according to the generalised gamma-density can indeed lead to the path length statistics given by the MOZAIC data.

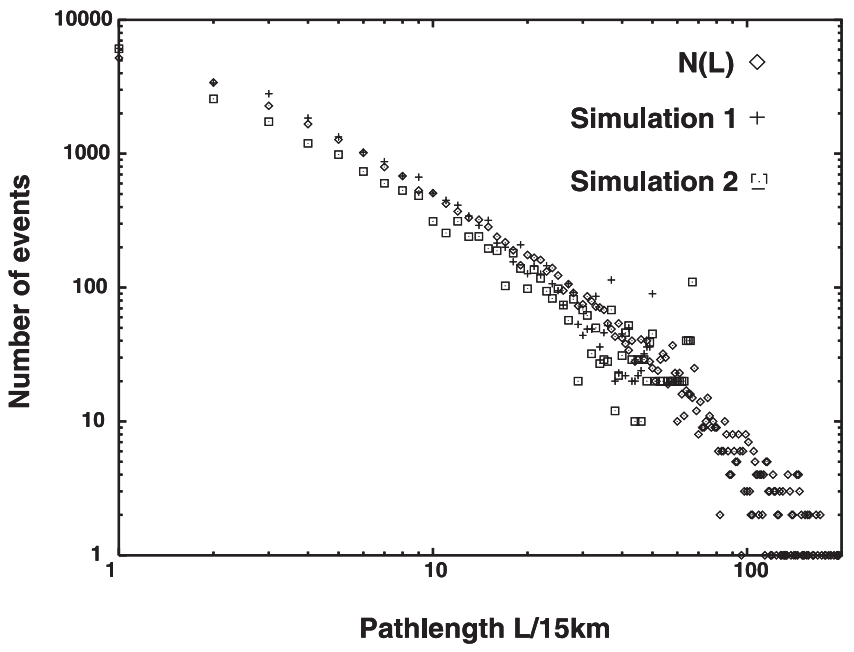

Fig. 5. Frequency distribution of path lengths inside ISSR according to two different numerical simulations using circular ISSRs with diameters distributed according to a generalised gamma-density, compared to the original data, $N(L)$

\subsection{Relevance of the result}

As stated there are so far no analytical methods to solve Eq. 6 for $h(D), f(L)$ given. Numerical methods to systematically invert the operator $T$ do not exist, because $T$ is nonlinear [i.e. $T\left[a h_{1}+b h_{2}\right] \neq a T\left[h_{1}\right]+b T\left[h_{2}\right]$, the reason for the nonlinearity is the appearance of $\langle D\rangle$ outside the integral, which is itself a functional of $h(D)]$. So far there are no mathematical methods to state whether a solution to Eq. (6) exists and if so, whether it is unique. This leaves our conclusion that there might be many more small ISSRs than apparent from the MOZAIC data uncertain, since we cannot be sure that the solution we found by trial and error is unique. In our solution, $f(L)$ came out as a generalised Meijer's Gfunction, which has a number of parameters whose meaning are not clear at all in the present context. A look at the table shows also that the computed variance is about $100 \mathrm{~km}$ larger than in the original data, which is certainly a weak point of our solution, but which can be an effect of the simplifying assumption of circular shape. In fact, the data $(N(L))$ can be fitted much more simply with a Weibull distribution, $f(L)=\gamma p L^{p-1} \exp \left(-\gamma L^{p}\right)$ with $\gamma=0.55$ and $p=0.5$, whose parameters can probably be interpreted more easily. However, we could not find any function $h(D)$ which makes $f(L)$ a Weibull distribution when applying Eq. 6 .

What have we learned from the exercise presented? There are only a couple of facts that we can accept from the measured path length distribution. First, there are very long paths (exceeding $1000 \mathrm{~km}$ ) which implies the existence of very large ISSRs. Second, most ISSRs do not reach such a size. On the other hand, the frequency distribution of path lengths is meaningless and its moments are otiose. Even in such a simplified case as considered here (circular ISSRs) we cannot determine uniquely and with certainty a "true" mean diameter of ISSRs from the path length data, and in the trial 
solution we found there is more than an order of magnitude difference between $\langle L\rangle$ and $\langle D\rangle$. Of course, the relation between $\langle L\rangle$ and a measure of size of ISSRs must be much more intricate in reality. Obviously, the true size distribution of ISSRs cannot be determined using commercial flight data. Instead we need dedicated research flights or advanced remote sensing methods for this purpose. Such an approach would also allow us to address the issue of different lifetimes of ISSRs of different sizes, which we could not consider in the present work.

\section{Conclusions}

We have presented the statistics of path lengths flown by MOZAIC aircraft inside ice-supersaturated regions (ISSRs) in the upper troposphere and lowermost stratosphere. The mean distance flown by MOZAIC aircraft within ice-supersaturated air was about $150 \mathrm{~km}$, which is consistent with earlier results of Detwiler and Pratt (1984) and with spatial scales of contrail clusters determined from satellite pictures (Bakan et al., 1994; Mannstein et al., 1999). The longest distance flown inside an ISSR extended for more than $3700 \mathrm{~km}$.

We considered then the possibility of a substantial selection bias in the path length data, caused by the obvious fact that the probability of crossing a certain ISSR by chance increases with the size of the ISSR. We performed a mathematical analysis of the problem, assuming for simplicity that all ISSR have circular shape. The resulting basic equation (Eq. 6) turned out to be not analytically nor numerically solvable with current mathematical methods. We found a solution, i.e. a "true" size distribution for the circular ISSRs by trial and error and tested it using two numerical simulations. The result was surprising because the mean "true" ISSR diameter was about 20 times smaller than the mean path length of the MOZAIC data. Whether the small ISSRs really do exist and how frequent they are is unknown, because we do not know whether our result is unique. In fact, there are at present no mathematical methods to state whether our basic equation has a solution at all, and if so, whether it is unique.

At least, our analysis demonstrates the possibility that there may be many more small ISSRs than is apparent from the path length data. One should be aware of this possibility when investigating ISSRs or other objects for which there are only path length data. The truth about shapes and sizes of ISSRs can only show up when dedicated research flights are conducted.

Acknowledgements. The authors thank Christoph Gugg and Heike Hoffmann of the Mathematics Institute of University of Augsburg for their advice on mathematical problems and Herrmann Mannstein (DLR) for his help in interpreting the satellite pictures of December 18, 1995. K. Gierens is supported by the Aerosolforschungsschwerpunkt (AFS) of the Bundesministerium für Bildung und Forschung. The project MOZAIC is funded by the Commission of the European Union and co-ordinated by Alain Marenco (Toulouse).

Topical Editor J.-P. Duvel thanks A. Heymsfield and O. Debarre for their help in evaluating this paper.

\section{References}

Bakan, S., M. Betancor, V. Gayler, and H. Graßl, Contrail frequency over Europe from NOAA-satellite images, Ann. Geophysicae, 12, 962-968, 1994.

Detwiler, A., and R. Pratt, Clear air seeding: opportunities and strategies, J. Weather Modif., 16, 46-60, 1984.

Gierens, K., U. Schumann, M. Helten, H. Smit, and A. Marenco, A distribution law for relative humidity in the upper troposphere and lower stratosphere derived from three years of MOZAIC measurements, Ann. Geophysicae, 17, 1218-1226, 1999.

Heymsfield, A. J., and L. M. Miloshevich, Homogeneous ice nucleation and supercooled liquid water in orographic wave clouds, J. Atmos. Sci., 50, 2335-2353, 1993.

Heymsfield, A. J., L. M. Miloshevich, C. Twohy, G. Sachse, and S. Oltmans, Upper-tropospheric relative humidity observations and implications for cirrus ice nucleation, Geophys. Res. Lett., 25, 1343-1346, 1998.

Mannstein, H., R. Meyer, and P. Wendling, Operational detection of contrails from NOAA-AVHRR-data, Int. J. Remote Sensing, 20, 1641-1660, 1999.

Marenco, A., V. Thouret, P. Nedelec, H. G. Smit, M. Helten, D. Kley, F. Karcher, P. Simon, K. Law, J. Pyle, G. Poschmann, R. Von Wrede, C. Hume, and T. Cook, Measurement of ozone and water vapour by Airbus in-service aircraft: the MOZAIC airborne program, an overview, J. Geophys. Res., 103, 2563125242, 1998.

Mathai, A. M., A handbook of generalized special functions for statistical and physical sciences, Clarendon Press, Oxford, 1993, 235pp., ISBN 0-19-853595-3.

Sassen, K., and G. C. Dodd, Haze particle nucleation simulation in cirrus clouds and applications for numerical and lidar studies, J. Atmos. Sci., 46, 3005-3014, 1989.

Ström, J., B. Strauss, T. Anderson, F. Schröder, J. Heintzenberg, and $\mathbf{P}$. Wendling, In situ observations of the microphysical properties of young cirrus clouds, J. Atmos. Sci., 54, 2542-2553, 1997. 